\title{
A!
}

This is an electronic reprint of the original article.

This reprint may differ from the original in pagination and typographic detail.

Fujikane, Masaki; Nagao, Shijo; Chrobak, Dariusz; Yokogawa, Toshiya; Nowak, Roman

\section{Room-Temperature Plasticity of a Nanosized GaN Crystal}

Published in:

Nano Letters

DOI:

10.1021/acs.nanolett.1c00773

Published: 11/08/2021

Document Version

Publisher's PDF, also known as Version of record

Published under the following license:

CC BY

Please cite the original version:

Fujikane, M., Nagao, S., Chrobak, D., Yokogawa, T., \& Nowak, R. (2021). Room-Temperature Plasticity of a Nanosized GaN Crystal. Nano Letters, 21(15), 6425-6431. https://doi.org/10.1021/acs.nanolett.1c00773

This material is protected by copyright and other intellectual property rights, and duplication or sale of all or part of any of the repository collections is not permitted, except that material may be duplicated by you for your research use or educational purposes in electronic or print form. You must obtain permission for any other use. Electronic or print copies may not be offered, whether for sale or otherwise to anyone who is not an authorised user. 


\title{
Room-Temperature Plasticity of a Nanosized GaN Crystal
}

\author{
Masaki Fujikane,* Shijo Nagao, Dariusz Chrobak, Toshiya Yokogawa, and Roman Nowak*
}

Cite This: Nano Lett. 2021, 21, 6425-6431

Read Online

ABSTRACT: GaN wurtzite crystal is commonly regarded as eminently brittle. However, our research demonstrates that nanodeconfined $\mathrm{GaN}$ compressed along the $\mathrm{M}$ direction begins to exhibit room-temperature plasticity, yielding a dislocation-free structure despite the occurrence of considerable, irreversible deformation. Our interest in M-oriented, strained GaN nanoobjects was sparked by the results of first-principles bandgap calculations, whereas subsequent nanomechanical tests and ultrahigh-voltage $(1250 \mathrm{kV})$ transmission electron microscopy observations confirmed the authenticity of the phenomenon. Moreover, identical experiments along the $\mathrm{C}$ direction produced only a quasi-brittle response. Precisely how this happens is

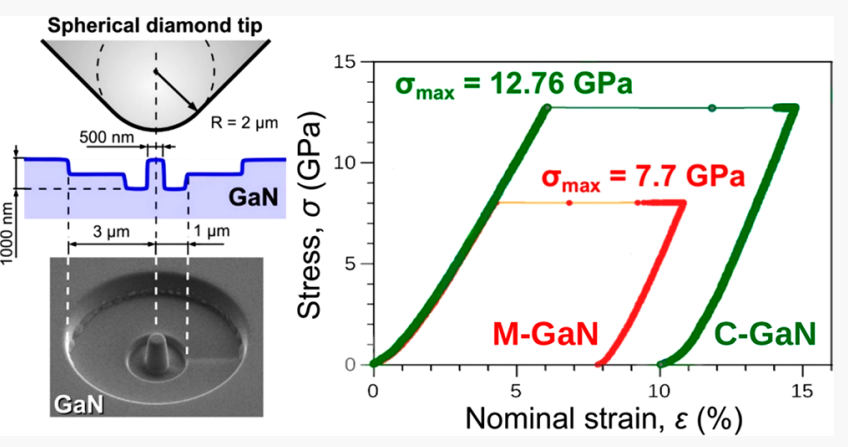
demonstrated by molecular dynamics simulations of the deformation of the C- and M-oriented GaN frustum, which mirror our nanopillar crystals.

KEYWORDS: GaN nanocrystals, nanoscale compression, plasticity, ultrahigh voltage electron microscopy, ab initio calculations, MD-simulations

emiconductors have been the life blood of technology ever $\checkmark$ since the first contact-point transistor was invented in 1947. ${ }^{1}$ It would seem therefore that every aspect of semiconducting materials has been thoroughly investigated, ${ }^{2}$ yet their mechanical properties have received significantly less attention than the optoelectronic ones. The majority of inorganic semiconductors are stiff, barely deformable solids with powerful interatomic bonding reflected by high melting temperatures $T_{\mathrm{m}} \cdot{ }^{3}$ However, more recent research has challenged that idea with the synthesis of a first ever plastic $\mathrm{Ag}_{2} \mathrm{~S}$ inorganic semiconductor ${ }^{4}$ or the discovery of plasticity of $\mathrm{ZnS}$ crystals under complete darkness, ${ }^{5}$ whereas the hightemperature mechanical behavior of ceramic and semiconductor nano-objects has been attracting increasing attention of the scientific world. ${ }^{6}$ Our own findings show that even a brittle semiconductor such as $\mathrm{GaN}$ can, under appropriate conditions (a nanodeconfined state ${ }^{7,8}$ and straining in a particular direction), display distinct plasticity even at room temperature (RT). At the same time, Kamimura, Kirchner, and Suzuki ${ }^{9}$ have estimated that GaN's transition from brittle to ductile would require a temperature of $800{ }^{\circ} \mathrm{C}$, suggesting that any talk of GaN's plastic behavior would be possible only in the context of high temperatures. It is little wonder then that GaN's plastic deformation at room temperature is utterly expected. This is also a reason why $\mathrm{GaN}$ plasticity has previously been methodically examined exclusively in high temperatures. ${ }^{10,11}$

$\mathrm{GaN}$ crystal has a well-established place in modern technology. ${ }^{12,13}$ In bulk mode and room temperature, GaN has long been known to display considerable strength, hardness, and brittleness. ${ }^{14-17}$ The same applies to GaN nano-objects that Huang et al. ${ }^{18}$ characterized as displaying only limited local plasticity in contrast to the "global" one exhibited by metallic micro-objects (cf. studies by $\mathrm{Nix}^{19}$ Minor, ${ }^{20}$ or Schuh ${ }^{21}$ and their research teams). Despite wideranging testing conditions, ${ }^{18,22-26}$ no such "global GaN plastic behavior" has been detected, yet our Letter proposes that it is real. We turn therefore to $\mathrm{GaN}$ crystal, whose structureunlike that of other semiconductors, e.g., $\mathrm{GaAs}^{27,28}$ or $\mathrm{Si}^{29}-$ has been shown to remain stable until the pressure reaches 47-60 GPa. ${ }^{30-33}$

We began by performing density functional theory (DFT) calculations of the bandgap $E_{\mathrm{g}}$ in a stressed $\mathrm{GaN}$ structure (Supporting Information A-1), using the Quantum Espresso software package. ${ }^{34,35}$ The exchange-correlation energy was determined according to the Perdew-Burke-Ernzerhof functional. ${ }^{36}$ The ultrasoft $\mathrm{Ga}$ and $\mathrm{N}$ pseudopotentials were selected from the PSLibrary database. ${ }^{37}$ The energy cutoff of $60 \mathrm{Ry}$ was established as the threshold for the wave function expansion, whereas the first Brillouin zone was sampled by

Received: February 23, 2021

Revised: July 16, 2021

Published: July 27, 2021 
applying the $11 \times 11 \times 11$ Monkhorst-Pack $k$-point mesh ${ }^{38}$ (Supporting Information A-1 and Figure S1).

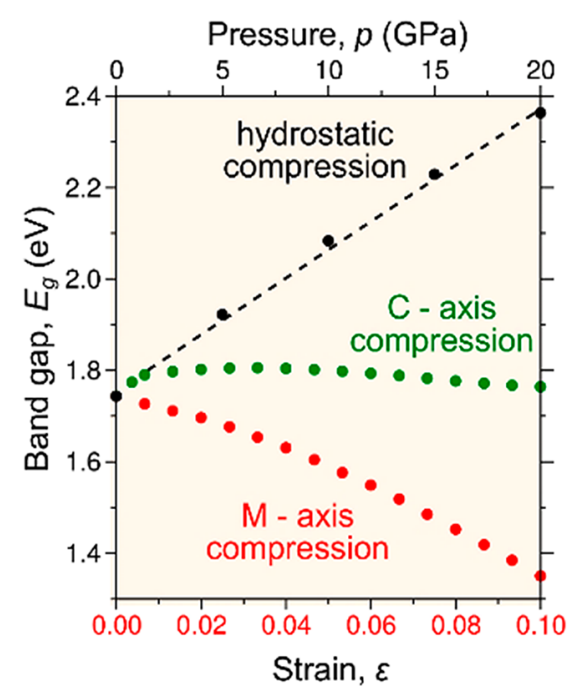

Figure 1. Stress-dependent changes of GaN bandgap deduced from $\mathrm{ab}$ initio calculations. The black dotted line (the upper pressure scale) concerns hydrostatic compression of GaN structure; the red and green data relate to the crystal compression (red scale refers to uniaxial strain) along the $\mathrm{M}[10 \overline{1} 0]$ and $\mathrm{C}[0001]$ directions, respectively.

The obtained quasi-linear rise of $E_{\mathrm{g}}$ with increasing hydrostatic pressure $p$ (Figure 1) agreed with earlier experimental observations, ${ }^{39-42}$ lending credibility to our calculations (see Supporting Information B). However, the declining $E_{\mathrm{g}}-\varepsilon$ relationship found for $\mathrm{GaN}$ when compressed along the $M[10 \overline{1} 0]$ direction (marked in red) was quite unexpected, diverging both from the hydrostatic (marked in black) and the C[0001]-axis stressing (marked in green). It implies a stark contrast in GaN's mechanical response depending on whether it is stressed along the $\mathrm{M}$ - or C-axis (Figure 1), where earlier reports ${ }^{13,30,43}$ have considered its elastic anisotropy as insignificant.

Topical experiments by Porowski et al. ${ }^{44}$ show the GaN bandgap commensurate with its melting temperature, and an increasing $T_{\mathrm{m}}-p$ dependence. Because the $T_{\mathrm{m}}$ value reflects crystal cohesion (Supporting Information B), it is reasonable to expect crystal "weakening" during straining along the $M$ direction (Figure 1). This significant loss of strength in $\mathrm{GaN}$ persuaded us to undertake an experimental assessment of its nanoscale deformation along the $M$ direction, which, to the best of our knowledge, had never been attempted before.

Consequently, GaN wafers $(5 \times 5 \times 0.4 \mathrm{~mm})$ with the $\mathrm{C}(0001)$ and $\mathrm{M}[10 \overline{1} 0]$ oriented surfaces were cut from a larger crystal grown by hydride vapor phase epitaxy in a way to avoid defect generation. An initial cathode luminescence examination of the materials confirmed their high quality: the threading dislocation density in $\mathrm{C}$ - and $\mathrm{M}$-oriented wafers equaled 1 $\times 10^{10}$ and $4 \times 10^{9} \mathrm{~m}^{-2}$, respectively. A set of virtually identical $\mathrm{GaN}$ nanopillars was carved in each of the prepared wafers using a two-stage focused ion beam (FIB) milling (Supporting Information A-2 and Figure S2), taking every precaution the FIB did not introduce dislocations in the fabricated items.

The RT nanocompression tests followed the approach developed by Schuh and his team ${ }^{21}$ as well as the updated standards for GaN nano-objects reviewed by Fatahilah et al. ${ }^{45}$
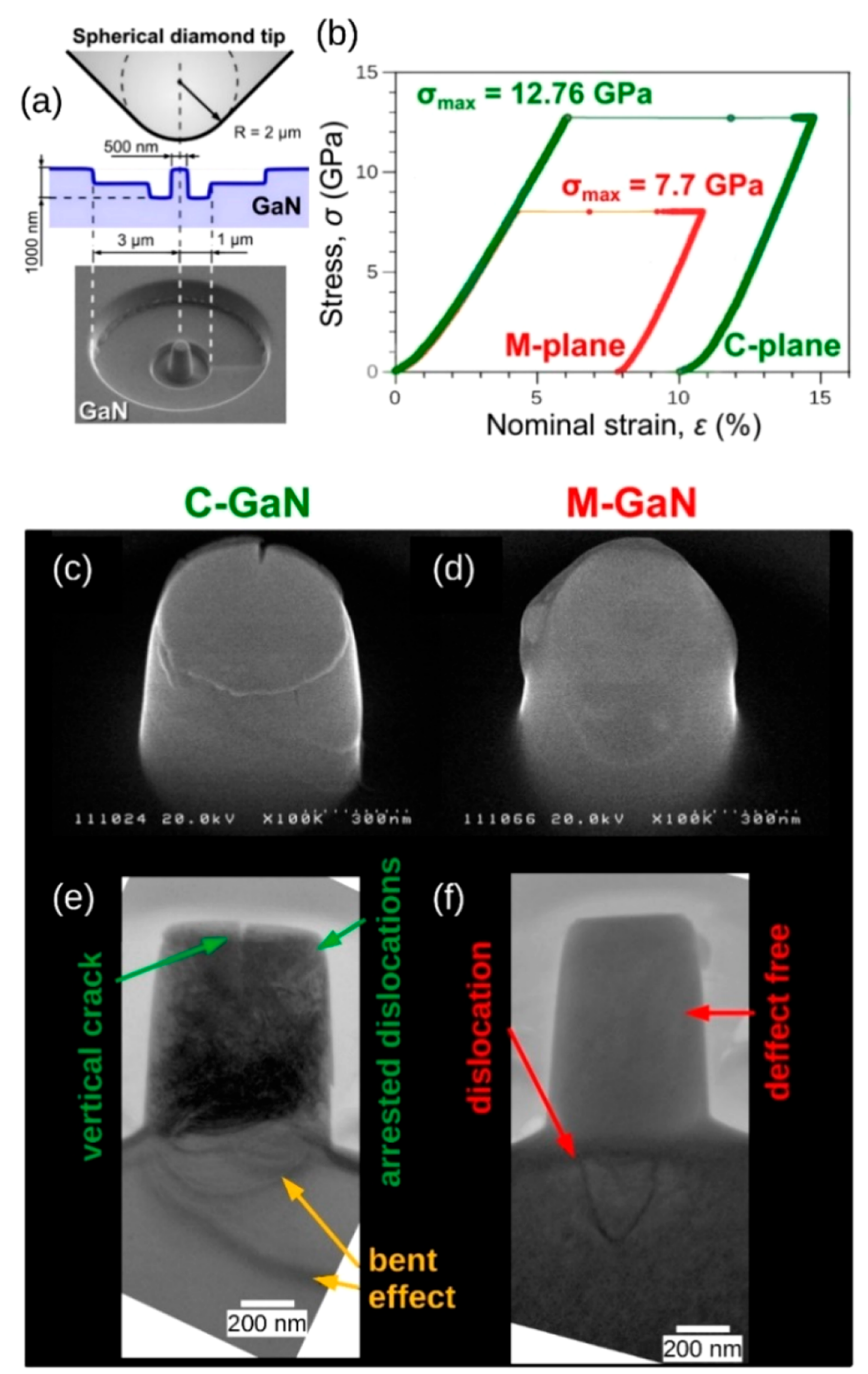

Figure 2. Results of nanocompression experiments carried out on the $\mathrm{C}$ - and M-oriented GaN nanopillars: (a) a schematic of the performed test, (b) the stress-strain $\sigma-\epsilon$ curves determined for the C-GaN and M-GaN nanopillars, the general SEM views of the postdeformed (c) $\mathrm{C}-\mathrm{GaN}$ and (d) M-GaN crystals. Also included are bright-field seethough views of the entire (e) C-GaN and (f) M-GaN pillar structure that remains after the single strain-burst deformation. (e) The Coriented $\mathrm{GaN}$ reveals a significant accumulation of stacked dislocations as against the $\mathrm{M}-\mathrm{GaN}$, which manifests a defect-free crystalline structure. The displayed quasi-brittle response of C-GaN with (c, e) a vertical crack formation contrasts with the plasticity evident in $(\mathrm{d}, \mathrm{f})$ the $\mathrm{M}-\mathrm{GaN}$. (See also the structure observed for inclined nanopillars in Figures S7 and S8.)

The experiments were carried out using a nanoindenter with precise test-geometry (Figure $2 \mathrm{a}$ ), by compressing each pillar to a different force-limit in an effort to elicit a single strainburst response (Supporting Information A-3 and Figure S6). This strategy proved successful, revealing an entire, irreversible deformation of certain $\mathrm{C}-\mathrm{GaN}$ and $\mathrm{M}-\mathrm{GaN}$ nanopillars accomplished under constant nominal stress $\sigma_{\max }$ (Figure 2b). The recorded stress-strain curves display a disparity between the M-GaN and C-GaN nano-objects, with displacement bursts occurring at different stress levels, namely, $\sigma_{\max }=$ 7.7 (red curve) and 12.7 GPa (green curve), respectively. The latter conforms to the RT hardness of $\mathrm{GaN}$ thick-films of $H \approx$ $12 \mathrm{GPa}^{13,14}$ leading to the conclusion that the $\mathrm{C}-\mathrm{GaN}$ case represents the commonly recognized mechanical properties of 


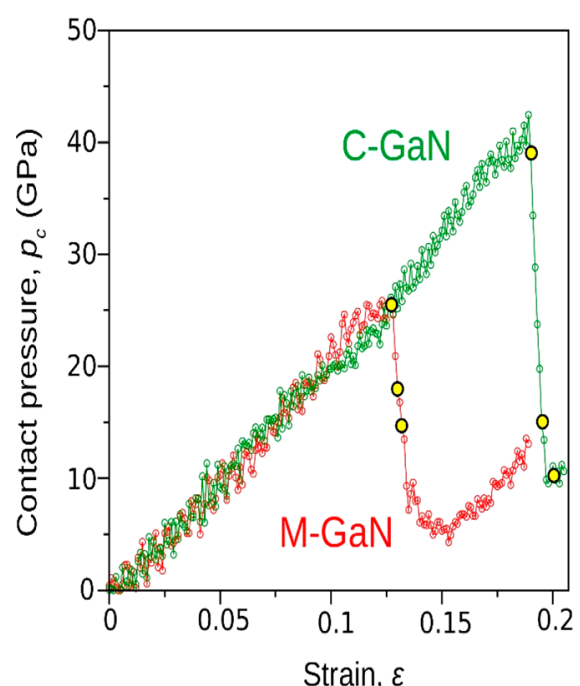

Figure 3. Contact pressure-strain $p_{\mathrm{c}}-\varepsilon$ relationships for the $\mathrm{C}$ - and $\mathrm{M}$-oriented $\mathrm{GaN}$ frustums compressed at $300 \mathrm{~K}$ show a difference in mechanical response of nano-objects. The abrupt pressure-drops in $p_{c}-\varepsilon$ graphs that correspond to a single-burst deformation (Figure $2 \mathrm{~b}$ ) confirm considerably stiffer behavior of the $\mathrm{C}-\mathrm{GaN}$, whereas the $\mathrm{M}$-oriented frustum starts to deform under significantly lower stress. A visualization of structure evolution under increasing strain $\varepsilon$ (selected stages of compression marked by yellow points) is presented in Figure 4.

GaN crystal, ${ }^{13-17,25,26}$ whereas the M-GaN one corroborates the "unexpectedly weak behavior" foreseen by ab initio calculations (Figure 1). Our claim is strengthened further by an inspection of nanopillars deformed according to a single strain-excursion pattern using scanning electron microscopy (SEM), which exposed the difference between a brittle-ceramic manner of the C-GaN deformation (Figure 2c) and plastic performance by the M-GaN (Figure 2d). Credible proof of this idiosyncrasy came from ultrahigh-voltage $(1250 \mathrm{kV})$ transmission electron microscopy (UHV-TEM), which enabled first-hand observation of GaN structure in our $500 \mathrm{~nm}$ thick nano-objects (Supporting Information C-1 and C-3 and Figure S7). The deformed C-GaN nanopillar contains a huge number of accumulated dislocations (Figure 2e) and a vertical crack, which confirms its quasi-brittle response. This is similar to the effect found for GaN nanowires by Huang et al. ${ }^{18}$ and the conventional view of $\mathrm{GaN}$ properties. ${ }^{13-17}$ Like the majority of earlier works on compressed GaN micropillars, they report a vertical crack, which is due to their exclusive concentration on C-oriented objects. ${ }^{22-24}$

By contrast, our results concern a defect-free M-GaN structure obtained after severe $\left(\varepsilon_{\mathrm{M}-\mathrm{GaN}}=8 \%\right)$ irreversible deformation (Figure 2b). As it happened, thorough microscopic observations of the whole volume of the M-oriented pillars variously inclined to the incident electron beam (see Figure S8) failed to detect a single dislocation. There is no doubt that, if any such defects in the nano-object structure existed, they would have left a trace in bright-field UHV-TEM images (Figure 2f), similarly to the defects arrested within the $\mathrm{C}-\mathrm{GaN}$ (Figure 2e). The absence of dislocations in the M-GaN pillar on the one hand, and their existence in the confined rootsubstrate (Figure $2 \mathrm{f}$ and Figure S8) on the other, is ample proof that dislocation activity did indeed occur, causing the hard GaN crystal to deform through slip. It bears emphasizing that the phenomenon we are dealing with differs from

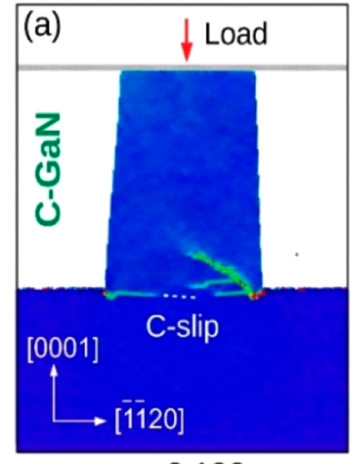

$\varepsilon=0.190$

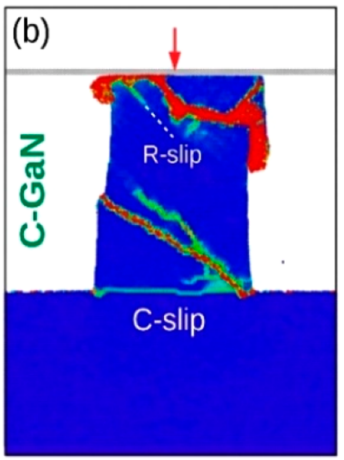

$\varepsilon=0.195$

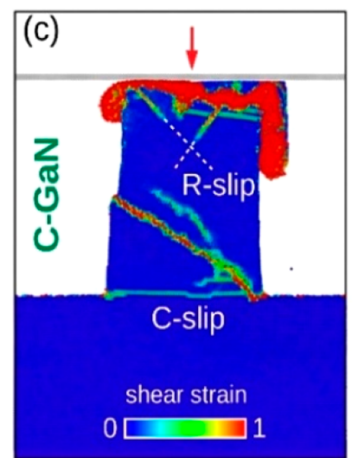

$\varepsilon=0.199$
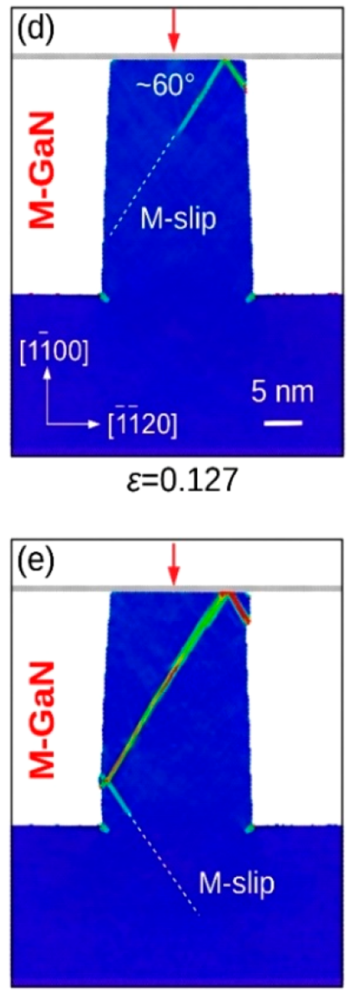

$\varepsilon=0.129$

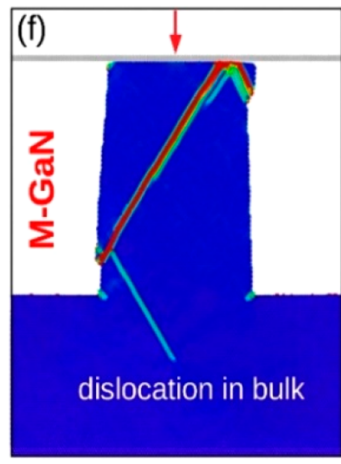

$\varepsilon=0.131$
Figure 4. Contrasting structure evolution during compression of the $(a-c) C$ - and $(d-f)$ M-oriented $\mathrm{GaN}$ frustums derived from our MD simulations. The sequence of selected strain values complies with the yellow points in $p_{c}-\varepsilon$ curves for both objects (Figure 3 ), whereas the depicted structural changes are exposed in the vertical, diameter crosssection of a modeled pillar. The atoms are marked in colors according to the atomic shear stress level determined in their actual location. $(\mathrm{a}-\mathrm{c})$ Massive accumulation of defects in the C-GaN frustum and $(\mathrm{d}-\mathrm{f})$ loss of its integrity due to crystal-block sliding and the extrusion of stressed material are in marked contrast to the plasticity of the MGaN realized by multiple $\mathrm{M}$ slips.

"dislocation starvation" or "mechanical annealing" reported for metallic nano-objects, ${ }^{19,20,46}$ concerning as it does a strong solid with considerable resistance to dislocations motion. ${ }^{16}$

We have tried to account for the experimental data using MD simulations. Our computations were performed with the LAMMPS code $^{47}$ for two frustum $\left(\Phi_{\text {top }} \times H \times \Phi_{\text {bottom }}\right)$ objects: C-GaN $(14.7 \times 29.8 \times 17.9 \mathrm{~nm})$ and M-GaN $(14.9 \times$ $29.9 \times 17.7 \mathrm{~nm}$ ) placed on a GaN wafer, which reflect the geometry of the examined samples. The interactions among the atoms within the wurtzite structure were described using 

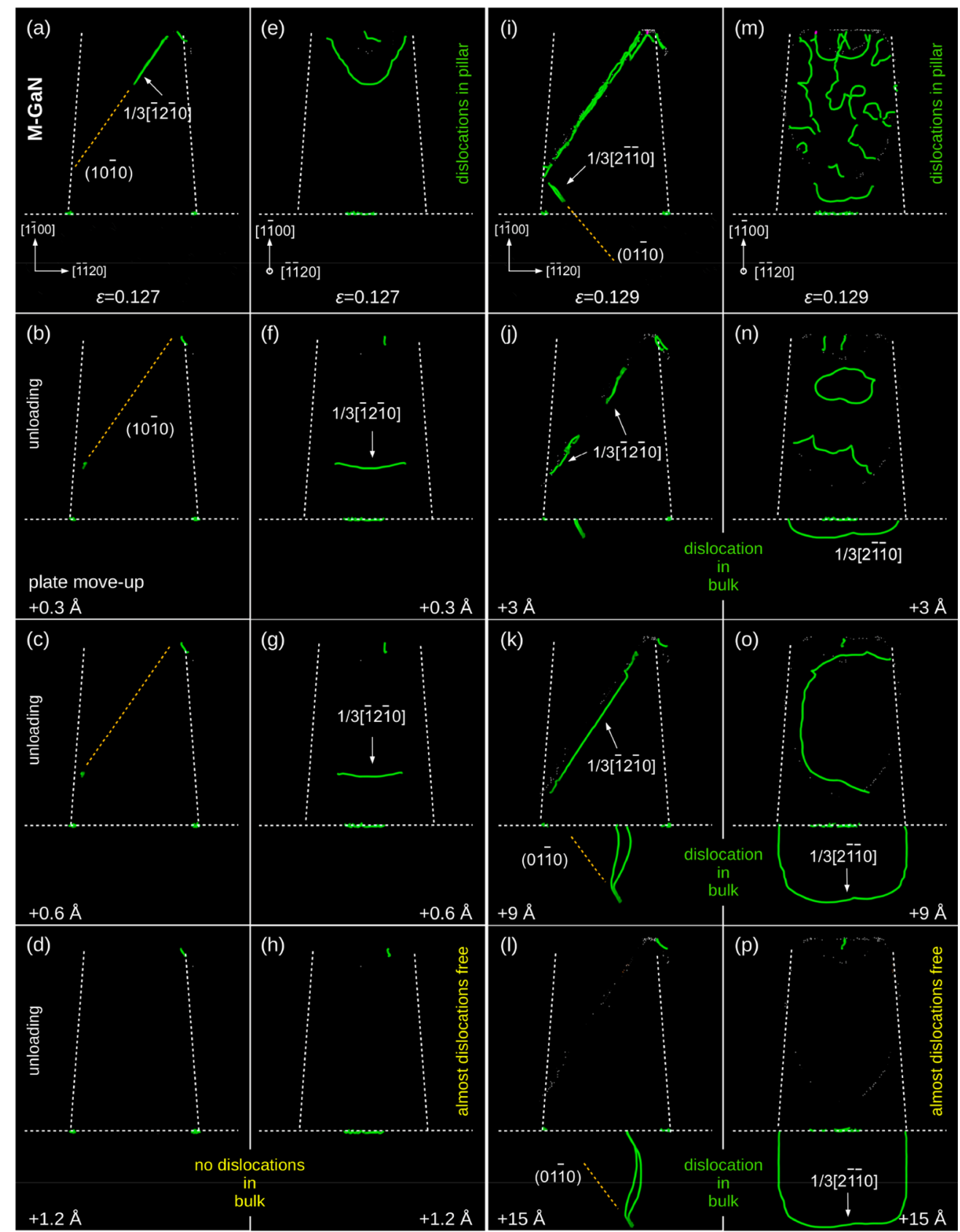

Figure 5. DXA visualization of dislocations in the M-GaN frustum (a, e) strained up to $\varepsilon=0.127$ and $(\mathrm{b}-\mathrm{h})$ after unloading. Panels a and e concern the pillar viewed along two different, perpendicular directions. Similarly, in the M-GaN frustum (i, $\mathrm{m}$ ) prestrained up to $\varepsilon=0.129$ and ( $\mathrm{j}-$ p) unloaded. $(a-h)$ Significantly, the dislocation induced during loading disappears on reaching the lateral surface during unloading. However, in the larger prestrained frustum $(\mathrm{i})$ and $(\mathrm{m}),(\mathrm{i}-\mathrm{p})$ the relaxation process results in the annihilation of defects except for a single one $(1$ and $\mathrm{p})$ that extends into the M-oriented root substrate. The additional description of unloading stages for differently strained M-GaN is provided in Supporting Information D and Figure S5.

the Stillinger-Weber ${ }^{48}$ potential created by Béré and Serra, ${ }^{49}$ who demonstrated its accuracy in modeling GaN lattice parameters and elastic constants (refer to Supporting Information A-4). The deformation path of each object was induced at $300 \mathrm{~K}$ by applying a load to the rigid, horizontal plate while in contact with the upper frustum surface. To achieve a quasi-static deformation, we relaxed the plate shift increments of $0.3 \AA$ within 2 ps time intervals (details in Supporting Information A-4).

The MD-simulated deformation history of C-GaN frustum displayed in the contact pressure-strain $\left(p_{c}-\varepsilon\right)$ curves (Figure 3) conforms qualitatively with the experimental data obtained for compressed GaN microprisms by Wheeler et al., ${ }^{24}$ or those by Huang et al. ${ }^{18}$ for GaN nanowires squeezed along their $\mathrm{C}[0001]$ axis. This agrees with a common perception of $\mathrm{GaN}$ objects as stiff and brittle materials in a range of testing temperatures. ${ }^{13,17}$ However, the simulated $p_{c}(\varepsilon)$ characteristics unveiled a noticeable difference in the mechanical conduct of the $\mathrm{C}$ - and $\mathrm{M}$-oriented frustums quite unlike the moderate directionality registered in our earlier nanoindentation experiments on bulk GaN crystal. ${ }^{25,26}$

The abrupt stress-drop recorded in both $p_{\mathrm{c}}(\varepsilon)$ relationships (Figure 3 ) corresponds to the strain bursts (Figure $2 \mathrm{~b}$ and Figure S6), as MD simulations commonly apply depth- 
controlled compression, whereas nanomechanical testing is load-controlled. ${ }^{7,50}$ It turns out that the $\mathrm{C}$-GaN requires a significantly higher contact pressure than the $\mathrm{M}-\mathrm{GaN}$ to initiate irreversible deformation (Figure 3), which accords with our experiments (Figure 2b). However, neither the simulated nor measured mechanical characteristics of $\mathrm{C}$ - and $\mathrm{M}$-oriented nano-objects (Figure $2 \mathrm{~b}$ and 3 ) appear to resolve the dilemma we are in, namely, of a sizable plastic $\mathrm{M}-\mathrm{GaN}$ deformation with a resulting intact crystalline structure on the one hand, and a quasi-brittle response of the $\mathrm{C}-\mathrm{GaN}$ with arrested dislocations (Figure 2) on the other.

The answer was provided by a visualization of the evolution of a strained atomic GaN structure recently made available with OVITO (Open Visualization Tool) and the dislocation extraction algorithm (DXA). ${ }^{51}$ In particular, our employment of the atomic shear strain modifier enabled us to envision the shear strains that atoms are subjected to and determine active slip planes, existing dislocations, and their Burgers vectors ${ }^{52}$ (see Supporting Information D-1). We found that the onset of an irreversible C-GaN deformation (Figure 3) concerns defect activity at the lower end of the modeled pillar (refer to Figure 4a). It involves limited local, quasi-elastic movement of dislocation lines on the $\mathrm{C}$ planes (DXA-details in Supporting Information D and Figure S3) that leads to accumulation of defects inside the crystal. The irreversible deformation starts with dislocation generation at the base of the frustum (Figure S3) despite the inevitable presence of higher stress close to its upper end (smaller cross-section area). We contend that the loading of a significantly strong and stiff $\mathrm{C}-\mathrm{GaN}$ nanopillar results in a moderate stress concentration along the perimeter of the bottom contact and local change in the GaN lattice orientation.

As deformation proceeds, the $\mathrm{R}$ slip operates in the upper part of the nano-object and forms a typical "mushroom profile" of extruded material close to the contact (Figures $4 b, c$ ), similarly to the observations by Huang et al. ${ }^{18}$ In reality, stress relaxation is realized by outward movement of the material immediately below the squeezing tool (with only a limited contribution from the $\mathrm{C}$ and $\mathrm{R}$ slip) and by vertical cracking of the $\mathrm{C}-\mathrm{GaN}$ pillar which both we (Figure 2c, e) and other authors ${ }^{22-24}$ have observed. The lion's share of the dislocations generated in the $\mathrm{C}-\mathrm{GaN}$ frustum is stacked in the crystal volume, as demonstrated by our simulations (Figure $4 a-c)$ and UHV-TEM observations (Figure 2e), because they are unable to escape the pillar volume or slip either during deformation or unloading (Supporting Information Figure S4).

The visualization highlights the plastic response of the $\mathrm{M}$ $\mathrm{GaN}$ (Figures $4 \mathrm{~d}-\mathrm{f}$ and 3 ) in marked contrast to the vast defect accumulation in the compressed C-GaN (Figure $4 a-c$ ). This kind of C-GaN's "quasi-brittle" behavior is consistent with the common perception of the mechanical properties of our nitride. $^{13-17,30,43}$ Particularly revealing, however, is the unobstructed, well-defined, multiple dislocation slip on the $\mathrm{M}$ planes right across the M-GaN crystal (Figure 4d, e), with some of it entering the substrate area (Figure 4f), which our TEM experiments had detected (Figure 2f). Indeed, the Peierls-Nabarro stress for the GaN prismatic $M\langle 11 \overline{2} 0\rangle\{1 \overline{1} 00\}$ slip, claimed by Kamimura et al. ${ }^{53}$ as well as Yonenaga and Motoki $^{54}$ to be lower than the Peierls barrier for other slip systems, indicates the possibility of the M slip.

One final piece of the puzzle was missing: why should the above mechanism result in a dislocation-free M-GaN structure (Figure 2f) despite the sizable plastic deformation (Figure 2b)?
Our MD simulations of the loading path (Figure 4) showed that, similarly to the $\mathrm{C}-\mathrm{GaN}$, the M-oriented frustum initially also contains dislocations, although to a much lesser degree (compare Figure $4 d-f$ and Figure $4 a-c$ ). In search for the answer, we investigated the evolution of defects during the unloading of the $\mathrm{M}-\mathrm{GaN}$. The DXA revealed that the generated dislocations (Figure $4 \mathrm{~d}-\mathrm{f}$ ) did not in fact contract during unloading (Figure 5). Instead, they expand and annihilate themselves on the lateral surface (Figure $5 b, c, f$, g), leaving behind a perfect $\mathrm{GaN}$ structure (details in Supporting Information D-2 and Figure S5), in full confirmation of our UHV-TEM observations (Figure 2f). Some other dislocations located close to the bottom of the frustum (Figure $5 \mathrm{i}, \mathrm{m}$ ) enter the substrate (Figure $5 \mathrm{j}-1$ and Figure $5 n-p)$ during unloading, again in accordance with our experiments (Figure 2f). Taken together, both our experiments and simulations stipulate that a specifically oriented GaN nanopillar will not perform like a brittle material.

Contemporary developments in $\mathrm{GaN}$ fabrication ${ }^{55}$ are opening the way to appliances capable of outperforming $\mathrm{Si}$ based products. They include nanocolumn LEDs, ${ }^{13}$ the next generation of power-electronic devices, or wirelessly powered systems in autonomous cars. ${ }^{56,57}$ Consequently, we are witnessing increased demand for all-embracing knowledge of the mechanical properties of GaN nanovolumes. ${ }^{58}$ The plastic response of $\mathrm{M}$-oriented $\mathrm{GaN}$ nano-objects goes some way toward meeting that demand.

\section{ASSOCIATED CONTENT}

\section{Supporting Information}

The Supporting Information is available free of charge at https://pubs.acs.org/doi/10.1021/acs.nanolett.1c00773.

Details of the numerical and experimental methods; discussion on a stress-dependent bendgap-melting temperature $E_{\mathrm{g}}-T_{\mathrm{m}}$ relationship for $\mathrm{GaN}$; additional experimental particulars; (D) MD-based analysis (Figures S3-S5) of GaN nanoscale deformation; (E) supplementary references; and Figures $\mathrm{S} 1-\mathrm{S} 8$ (PDF)

\section{AUTHOR INFORMATION}

\section{Corresponding Authors}

Masaki Fujikane - Applied Materials Technology Center, Technology Division, Panasonic Corporation, Kyoto 6190237, Japan; Email: fujikane.masaki@jp.panasonic.com Roman Nowak - Institute of Scientific and Industrial Research, Osaka University, Osaka 567-0047, Japan; Extreme Energy-Density Research Institute, Nagaoka University of Technology, Nagaoka, Niigata 940-2188, Japan; Nordic Hysitron Laboratory, School of Chemical Engineering, Aalto University, Aalto 00076, Finland; (1) orcid.org/0000-0002-2708-7375;

Email: roman.nowak@aalto.fi

\section{Authors}

Shijo Nagao - Institute of Scientific and Industrial Research, Osaka University, Osaka 567-0047, Japan

Dariusz Chrobak - Extreme Energy-Density Research Institute, Nagaoka University of Technology, Nagaoka, Niigata 940-2188, Japan; Present Address: Institute of Materials Engineering, University of Silesia in Katowice, 40-500 Chorzów, Poland 
Toshiya Yokogawa - Opto-Energy Research Center, Depatment of Materials Science \& Engineering, Yamaguchi University, Yamaguchi 755-8611, Japan

Complete contact information is available at: https://pubs.acs.org/10.1021/acs.nanolett.1c00773

\section{Author Contributions}

M.F., S.N., T.Y., and R.N. conceived the original idea. M.F. and S.N. performed nanocompression experiments. S.N. carried out initial MD simulations, whereas M.F. was involved in FIB carving of nanopillars as well as UHV-TEM observations. D.C. accomplished advanced MD simulations of structural changes in deformed $\mathrm{GaN}$ nanocrystals, identified dislocations and explained their movement to root substrate in M-GaN. T.Y. and R.N. supervised the research and analyzed the data. R.N. wrote the manuscript with contributions of all authors, who have given approval to its final version.

\section{Funding}

This research was sponsored mainly by the Panasonic Corp., Japan, as part of their cooperation with Japanese universities. Nagaoka University of Technology and Osaka University were cosponsors of short-term "visiting scholar" positions. The Aalto University offered a part of the computational resources.

\section{Notes}

The authors declare no competing financial interest.

\section{ACKNOWLEDGMENTS}

We gratefully acknowledge Prof. Shuji Nakamura (University of California, Santa Barbara) for his invaluable criticism, stimulating comments. and important suggestions. Equally appreciated are the observations and remarks by Prof. William D. Nix (Stanford University). R.N. acknowledges invaluable discussions with Prof. Toshihiro Shimada (Hokkaido University) and his role in ascertaining the quality of the nanopillars used in our experiments. R.N. also expresses appreciation to Prof Koichi Niihara and Prof. Hisayuki Suematsu (Nagaoka University of Technology) as well as to Mr. Michael Berg (Bruker Nano Surfaces, Los Angeles) for their long-standing support and many an eye-opener. MF is thankful to Mr. Hideki Hata and Tomohiko Ueda (Panasonic Corp.) for rewarding discussions concerning TEM observations. Finally, R.N. and D.C. acknowledge the support from Osaka University and Nagaoka University of Technology during their time as guest-scholars. This research was sponsored by Panasonic Corp., Japan, as part of cooperation with Japanese universities.

\section{ABBREVIATIONS}

MD, Molecular Dynamics; UHV-TEM, ultrahigh voltage transmission microscopy; DXA, dislocation extraction algorithm

\section{REFERENCES}

(1) Bardeen, J.; Brattain, W. The transistor, a semi-conductor triode. Phys. Rev. 1948, 74, 230-231.

(2) Shen, G.; Fan, Z. Flexible Electronics: From Materials to Devices; World Scientific, 2016.

(3) Gilman, J. J. Chemistry and Physics of Mechanical Hardness; John Willey \& Sons, 2009.

(4) Shi, X.; Chen, H.; Hao, F.; Liu, R.; Wang, T.; Qiu, P.; Burkhardt, U.; Grin, Y.; Chen, L. Room-temperature ductile inorganic semiconductor. Nat. Mater. 2018, 17, 421-426.
(5) Oshima, Y.; Nakamura, A.; Matsunaga, K. Extraordinary plasticity of an inorganic semiconductor in darkness. Science 2018, 360, 772-774.

(6) Grosso, R. L.; et al. In situ transmission electron microscopy for ultrahigh temperature mechanical testing of $\mathrm{ZrO}_{2}$. Nano Lett. 2020, 20, 1041-1046.

(7) Chrobak, D.; Tymiak, N.; Beaber, A.; Ugurlu, O.; Gerberich, W. W.; Nowak, R. Deconfinement leads to changes in the nanoscale plasticity of silicon. Nat. Nanotechnol. 2011, 6, 480-484.

(8) Cross, G. L. W. Silicon nanoparticles: Isolation leads to change. Nat. Nanotechnol. 2011, 6, 467-468.

(9) Kamimura, Y.; Kirchner, H. O. K.; Suzuki, T. Yield strength and brittle-to-ductile transition of boron-nitride and gallium-nitride. Scr. Mater. 1999, 41, 583-587.

(10) Wheeler, J. M.; et al. Extraction of plasticity parameters of GaN with high temperature, in situ micro-compression. Int. J. Plast. 2013, 40, 140-151.

(11) Maniatty, A.; Karvani, P. Constitutive relations for modelling single crystal $\mathrm{GaN}$ at elevated temperatures. J. Eng. Mater. Technol. 2015, 137 (011002), 1-7.

(12) Nanishi, Y. Nobel Prize in Physics: The birth of the blue LED. Nat. Photonics 2014, 8, 884-886.

(13) Morkoç, H. Nitride Semiconductor Devices: Fundamentals and Applications; Wiley-VCH: Weinheim, Germany, 2013.

(14) Drory, M. D.; Ager, J. W., III; Suski, T.; Grzegory, I.; Porowski, S. Hardness and fracture toughness of bulk single crystal gallium nitride. Appl. Phys. Lett. 1996, 69, 4044-4046.

(15) Nowak, R.; et al. Elastic and plastic properties of GaN determined by nanoindentation of bulk crystal. Appl. Phys. Lett. 1999, 75, 2070-2072.

(16) Yonenaga, I. Strength, dislocation mobility, and photoluminescence of plastically deformed GaN. Trends Cond. Matter Phys. Res. 2006, 61-76.

(17) Cheng, Y.; Cai, D.; Wang, H.; Wu, J.; Liu, X.; Zhang, G.; Yu, T. Anisotropic fracture toughness of bulk GaN. Phys. Status Solidi B 2018, 255, 1700515.

(18) Huang, J. Y.; Zheng, H.; Mao, S. X.; Li, Q.; Wang, G. T. In situ nanomechanics of GaN nanowires. Nano Lett. 2011, 11, 1618-1622.

(19) Nix, W. D.; Lee, S.-W. Micro-pillar plasticity controlled by dislocation nucleation at surfaces. Philos. Mag. 2011, 91, 1084-1096.

(20) Shan, Z. W.; Mishra, R. K.; Syed Asif, S. A.; Warren, O. L.; Minor, A. M. Mechanical annealing and source-limited deformation in submicrometre-diameter Ni crystals. Nat. Mater. 2008, 7, 115-119.

(21) Lai, A.; Du, Z.; Gan, C. L.; Schuh, C. A. Shape memory and superplastic ceramics at small scales. Science 2013, 341, 1505-1508.

(22) Kang, S.-H.; Fang, T.-H. Size effect on compression properties of $\mathrm{GaN}$ nanocones examined using in situ transmission electron microscopy. J. Alloys Compd. 2014, 597, 72-78.

(23) Sung, T. H.; Huang, J. C.; Hsu, J. H.; Jian, S. R. Mechanical response of GaN film and micropillar under nanoindentation and microcompression. Appl. Phys. Lett. 2010, 97, 171904.

(24) Wheeler, J. M.; Niederberger, C.; Tessarek, C.; Christiansen, S.; Michler, J. Extraction of plasticity parameters of $\mathrm{GaN}$ with high temperature, in situ micro-compression. Int. J. Plast. 2013, 40, 140151.

(25) Fujikane, M.; Inoue, A.; Yokogawa, T.; Nagao, S.; Nowak, R. Mechanical properties characterization of c-plane (0001) and m-plane (10-10) GaN by nanoindentation. Phys. Status Solidi C 2010, C7, $1798-1800$.

(26) Fujikane, M.; Yokogawa, T.; Nagao, S.; Nowak, R. Nanoindentation study on insight of plasticity related to dislocation density and crystal orientation in GaN. Appl. Phys. Lett. 2012, 101, 201901.

(27) Nowak, R.; Chrobak, D.; Nagao, S.; Vodnick, D.; Berg, M.; Tukiainen, A.; Pessa, M. An electric current spike linked to nanoscale plasticity. Nat. Nanotechnol. 2009, 4, 287-291.

(28) Chrobak, D.; Nordlund, K.; Nowak, R. Non-dislocation origin of GaAs nanoindentation pop-in event. Phys. Rev. Lett. 2007, 98, 045502 . 
(29) Abram, R.; Chrobak, D.; Nowak, R. Origin of a nanoindentation pop-in event in silicon crystal. Phys. Rev. Lett. 2017, 118, 095502.

(30) Usman, Z.; et al. Structural, elastic constant, and vibrational properties of wurtzite gallium nitride: A first principle approach. $J$. Phys. Chem. A 2011, 115, 14502-14509.

(31) Perlin, P.; Jauberthie-Carillon, C.; Itie, J. P.; San Miguel, A.; Grzegory, I.; Polian, A. Raman scattering and x-ray-absorption spectroscopy in gallium nitride under high pressure. Phys. Rev. B: Condens. Matter Mater. Phys. 1992, 45, 83-89.

(32) Mata, R.; Cros, A.; Hestroffer, K.; Daudin, B. Surface optical phonon modes in GaN nanowire arrays: Dependence on nanowire density and diameter. Phys. Rev. B: Condens. Matter Mater. Phys. 2012, $85,035322$.

(33) Caro, M. A.; Schulz, S.; O’Reilly, E. P. Hybrid functional study of the elastic and structural properties of wurtzite and zinc-blende group-III nitrides. Phys. Rev. B: Condens. Matter Mater. Phys. 2012, 86, 014117.

(34) Giannozzi, P.; et al. QUANTUM ESPRESSO: a modular and open-source software project for quantum simulations of materials. $J$. Phys.: Condens. Matter 2009, 21, 395502.

(35) Giannozzi, P.; et al. Advanced capabilities for materials modelling with QUANTUM ESPRESSO. J. Phys.: Condens. Matter 2017, 29, 465901.

(36) Perdew, J. P.; Burke, K.; Ernzerhof, M. Generalized gradient approximation made simple. Phys. Rev. Lett. 1996, 77, 3865.

(37) Dal Corso, A. Pseudopotentials periodic table: From $\mathrm{H}$ to $\mathrm{Pu}$. Comput. Mater. Sci. 2014, 95, 337-350.

(38) Monkhorst, H. J.; Pack, J. D. Special points for Brillouin-zone integrations. Phys. Rev. B 1976, 13, 5188-5192.

(39) Utsumi, W.; Saitoh, H.; Kaneko, H.; Watanuki, T.; Aoki, K.; Shimomura, O. Congruent melting of gallium nitride at $6 \mathrm{GPa}$ and its application to single-crystal growth. Nat. Mater. 2003, 2, 735-738.

(40) Wolff, G. A.; Toman, L.; Field, N. J.; Clark, J. C. Relationship of hardness, energy gap and melting point of diamond type and related structures. In Halbleiter und Phosphore/Semiconductors and Phosphors/ Semiconducteurs et Phosphores; Vieweg + Teubner Verlag: Wiesbaden, Germany, 1958; pp 463-470.

(41) Nag, B. R. An empirical relation between the melting point and the direct bandgap of semiconducting compounds. J. Electron. Mater. 1997, 26, 70-72.

(42) Manca, P. A relation between the binding energy and the bandgap energy in semiconductors of diamond or zinc-blende structure. J. Phys. Chem. Solids 1961, 20, 268-273.

(43) Wang, Z.; Zu, X.; Yang, L.; Gao, F.; Weber, W. J. Atomistic simulations of the size, orientation, and temperature dependence of tensile behavior in GaN nanowires. Phys. Rev. B: Condens. Matter Mater. Phys. 2007, 76, 045310.

(44) Porowski, S.; et al. The challenge of decomposition and melting of gallium nitride under high pressure and high temperature. J. Phys. Chem. Solids 2015, 85, 138-143.

(45) Fatahilah, M. F.; et al. Traceable nanomechanical metrology of GaN micropillar array. Adv. Eng. Mater. 2018, 20, 1800353.

(46) Greer, J. R.; De Hosson, J. T. M. Plasticity in small-sized metallic systems: Intrinsic versus extrinsic size effect. Prog. Mater. Sci. 2011, 56, 654-724.

(47) Plimpton, S. Fast parallel algorithms for short-range molecular dynamics. J. Comput. Phys. 1995, 117, 1-19.

(48) Stillinger, F. H.; Weber, T. A. Computer simulation of local order in condensed phases of silicon. Phys. Rev. B: Condens. Matter Mater. Phys. 1985, 31, 5262-5271.

(49) Béré, A.; Serra, A. Atomic structure of dislocation cores in GaN. Phys. Rev. B: Condens. Matter Mater. Phys. 2002, 65, 205323.

(50) Li, J.; Van Vliet, J.; Zhu, T.; Yip, S.; Suresh, S. Atomistic mechanisms governing elastic limit and incipient plasticity in crystals. Nature 2002, 418, 307-310.

(51) Stukowski, A. Visualization and analysis of atomistic simulation data with OVITO-the Open Visualization Tool Modelling. Modell. Simul. Mater. Sci. Eng. 2010, 18, 015012.
(52) Maras, E.; Trushin, O.; Stukowski, A.; Ala-Nissila, T.; Jónsson, $\mathrm{H}$. Global transition path search for dislocation formation in $\mathrm{Ge}$ on Si(001). Comput. Phys. Commun. 2016, 205, 13-21.

(53) Kamimura, Y.; Edagawa, K.; Takeuchi, S. Experimental evaluation of the Peierls stresses in a variety of crystals and their relation to the crystal structure. Acta Mater. 2013, 61, 294-309.

(54) Yonenaga, I.; Motoki, K. Yield strength and dislocation mobility in plastically deformed bulk single-crystal GaN. J. Appl. Phys. 2001, 90, 6539-6541.

(55) Xu, K.; Wang, J.-F.; Ren, G.-Q. Progress in bulk GaN growth. Chin. Phys. B 2015, 24, 066105.

(56) Parker, P. M. The 2019-2024 World Outlook for Gallium Nitride (GaN) Power Conversion Devices; ICON Group International Inc.: San Diego, 2018.

(57) Jamond, N. Energy harvesting efficiency in GaN nanowirebased nanogenerators: the critical influence of the Schottky nanocontact. Nanoscale 2017, 9, 4610-4619.

(58) Guo, J.; Qiu, C.; Zhu, H.; Wang, Y. Nanotribological properties of Ga- and N-faced bulk Gallium Nitride surfaces determined by nanoscratch experiments. Materials 2019, 12, 2653. 